This item was submitted to Loughborough's Research Repository by the author.

Items in Figshare are protected by copyright, with all rights reserved, unless otherwise indicated.

\title{
Energy replacement diminishes the postprandial triglyceride-lowering effect from accumulated walking in older women
}

PLEASE CITE THE PUBLISHED VERSION

https://doi.org/10.1007/s00394-020-02234-z

\section{PUBLISHER}

Springer (part of Springer Nature)

VERSION

AM (Accepted Manuscript)

\section{PUBLISHER STATEMENT}

This is a post-peer-review, pre-copyedit version of an article published in European Journal of Nutrition. The final authenticated version is available online at: https://doi.org/10.1007/s00394-020-02234-z.

\section{LICENCE}

CC BY-NC-ND 4.0

\section{REPOSITORY RECORD}

Miyashita, Masashi, Yuka Hamada, Kyoko Fujihira, Chihiro Nagayama, Masaki Takahashi, Stephen Burns, Alice Thackray, and David Stensel. 2020. "Energy Replacement Diminishes the Postprandial Triglyceridelowering Effect from Accumulated Walking in Older Women”. Loughborough University. https://hdl.handle.net/2134/12003309.v1. 
1 Energy replacement diminishes the postprandial triglyceride-lowering effect from

\section{2 accumulated walking in older women}

3

4 Masashi Miyashita $^{1}$, Yuka Hamada ${ }^{2,3}$, Kyoko Fujihira ${ }^{2,3}$, Chihiro Nagayama ${ }^{2}$, Masaki

5 Takahashi $^{4}$, Stephen F Burns ${ }^{5}$, Alice E Thackray ${ }^{6}$, David J Stensel ${ }^{6}$

6

$7 \quad{ }^{1}$ Faculty of Sport Sciences, Waseda University, Saitama, 359-1192, Japan

$8{ }^{2}$ Graduate School of Sport Sciences, Waseda University, Saitama, 359-1192, Japan

$9{ }^{3}$ Research Fellow of Japan Society for the Promotion of Science, Tokyo, 102-0083,

10 Japan

$11{ }^{4}$ Organization for University Research Initiatives, Waseda University, 139651,

12 Singapore

135 Physical Education and Sports Science Academic Group, National Institute of

14 Education, Nanyang Technological University, 637616, Singapore

$15{ }^{6}$ National Centre for Sport and Exercise Medicine, School of Sport, Exercise and

16 Health Sciences, Loughborough University, Leicestershire, LE11 3TU, United Kingdom

18 Address for Correspondence: 
19 Masashi Miyashita, Ph.D.

20 Waseda University

21 Faculty of Sport Sciences

22 2-579-15 Mikajima, Tokorozawa, Saitama, 359-1192, Japan

23 TEL/FAX: +81-4-2947-6774

24 E-mail: m.miyashita@waseda.jp 


\section{Abstract (250/150-250 words)}

26 [Purpose] Dietary replacement of an acute exercise-induced energy deficit offsets the

27 postprandial triglyceride (TG)-lowering effect of exercise in young boys and

28 middle-aged men. It is unclear whether these findings are observed when exercise is

29 accumulated in older adults. This study examined the effect of accumulating short bouts

30 of exercise, with and without dietary replacement of an exercise-induced energy deficit,

31 on postprandial TG in older women. [Methods] Seventeen older women ( $\geq 65$ years)

32 underwent three, 8-h trials: 1) control, 2) accumulated walking and 3) accumulated

33 walking with energy replacement. During the control trial, participants rested for $8 \mathrm{~h}$.

34 The accumulated walking trials comprised twenty 1.5 min brisk walking bouts

35 performed at a pre-determined self-selected pace separated by $15 \mathrm{~min}$ seated rest. In

36 each trial, participants consumed a standardised breakfast and lunch. The breakfast in

37 the accumulated walking with energy replacement trial included replacement of the

38 energy deficit $(0.62 \mathrm{MJ}, 149 \mathrm{kcal})$ induced by exercise. Venous blood samples were

39 collected fasted and at 2, 4, 6 and $8 \mathrm{~h}$ after breakfast. [Results] Time-averaged

40 postprandial serum TG concentrations over 8 hours were lower after accumulated

41 walking than control and accumulated walking with energy replacement (mean $\pm \mathrm{SD}$ :

$421.46 \pm 0.93$ vs $1.71 \pm 1.01$ vs $1.60 \pm 0.98 \mathrm{mmol} / \mathrm{L}$ respectively: main effect of trial $p=$ 
43 0.017). There was little difference between control and accumulated walking with

44 energy replacement. [Conclusions] Replacing the energy expenditure induced by

45 accumulating $30 \mathrm{~min}$ of brisk walking in short $(1.5 \mathrm{~min})$ bouts diminishes the

46 postprandial TG-lowering effect in older women.

47

$48 \quad$ Keywords

49 Energy replacement $\cdot$ Postprandial triglyceride $\cdot$ Lipid metabolism $\cdot$ Accumulated

50 walking $\cdot$ Older adults

52 Introduction

53 Repeated daily episodes of elevated non-fasting triglyceride (TG) and prolonged

54 residence in the circulation of TG-rich lipoproteins are a risk factor for cardiovascular

55 disease and all-cause mortality in men and women [1,2]. Thus, it is important to

56 consider lifestyle modifications which may be effective in reducing diurnal

57 exaggerations in postprandial TG.

59 Ample evidence supports the notion that an acute bout of aerobic exercise reduces

60 postprandial TG concentrations (for a review of relevant studies see [3]). Most 
61 randomised, cross-over studies in this area investigate participants whilst they are in a

62 state of energy deficit induced by exercise, which may at least partly mediate the

63 exercise-stimulated reduction in postprandial TG [3]. It is difficult to translate these

64 study findings to free living environments outside the laboratory where individuals with

65 normal free access to food and drink may replace the energy expended during exercise.

66 Thus, it is important to consider energy status when studying metabolic responses to

67 exercise [4].

69 To date, seven laboratory-based studies have examined the effects of an acute bout of

70 exercise, with and without dietary replacement of the exercise-induced energy deficit,

71 on postprandial TG concentrations with disparate findings [5-11]. Some studies note

72 that acute replacement of the energy deficit diminishes the exercise-induced reduction

73 in postprandial TG $[5-8,10,11]$ whereas others report lower postprandial TG

74 concentrations even after the energy expended during exercise was replaced

75 immediately after exercise [7, 9-11]. The amount of energy expended during exercise,

76 the intensity of exercise, and the type of replacement meal/drink are possible reasons for

77 the inconsistent findings among studies. Furthermore, most of the participants of these

78 seven previous studies were males ranging in age from adolescent boys to middle-aged 
79 men with only a few young, healthy adult women included in the analysis of two studies

80 alongside men [7, 11]. Investigations in older women ( $\geq 65$ years) represent an

81 important gap in current understanding considering excursions in TG concentrations

82 after meals are likely to be exaggerated in these individuals [12]. In addition, while

83 accumulated exercise can reduce postprandial TG concentrations in young adults [3],

84 more data for its effects are needed in older adults who may have a limited capacity to

85 perform exercise for long periods because of low fitness or pre-existing disease. Thus,

86 accumulated activity may be easier to incorporate into the daily life of older adults as

87 activities that are intermittent in nature often involve bouts lasting less than a few

88 minutes [13].

89

90 Therefore, the purpose of this study was to investigate the effect of accumulating short

91 bouts of brisk walking throughout the day, with and without dietary replacement of the

92 exercise-induced energy deficit, on postprandial TG in older ( $\geq 65$ years) women.

94 Methods

95 Participants

96 After approval from the Ethics Committee on Human Research of Waseda University 
97 (Approval number: 2017-103), 17 older women gave written informed consent to

98 participate in this study. Exclusion criteria were currently smoking, reporting a major

99 illness/disease, or physical problems (acute or chronic) limiting the ability to perform

100 activities of daily living. The physical characteristics of the participants are provided in

101 Table 1.

102

103

Insert Table 1 near here

104

105 Anthropometry

106 Body mass was measured to the nearest $0.1 \mathrm{~kg}$ using a digital scale (Inner Scan 50;

107 Tanita Corporation, Tokyo, Japan). Height was measured to the nearest $0.1 \mathrm{~cm}$ using a

108 wall-mounted stadiometer (YS-OA; AS ONE Corporation, Osaka, Japan). Body mass

109 index was calculated as weight in kilograms divided by the square of height in meters.

110 Waist circumference was measured to the nearest $0.1 \mathrm{~cm}$ at the level of the umbilicus

111 using a flexible plastic tape. Arterial blood pressure was measured from the right arm

112 after 5 min of seated rest by a standard mercury sphygmomanometer (605P; Yagami Co

113 Ltd, Yokohama, Japan). Two measurements were taken, and the mean of these values

114 was recorded. 


\section{Preliminary test}

117 After familiarisation with the treadmill (JOG NOW 700; Technogym, Milan, Italy),

118 each participant was asked to walk "briskly" for three minutes to determine their

119 walking speed and estimate the energy cost of the main exercise trial. "Brisk" walking

120 was defined as feeling slightly out of breath while walking but still able to hold a

121 conversation. Oxygen uptake, carbon dioxide production and respiratory exchange ratio

122 (RER) were measured breath-by-breath through a stationary gas analyser (Quark CPET,

123 COSMED, Rome, Italy). Energy expenditure was estimated from these using

124 standardised equations [14].

126 Standardisation of dietary intake and physical activity

127 Participants weighed and recorded all food and drink consumed the day before each trial

128 and refrained from drinking alcohol during this period. Participants replicated their

129 dietary intake from the first trial in all subsequent trials to ensure that dietary intake was

130 standardised across trials. Food diaries were analysed by a registered dietitian to

131 determine energy intake and macronutrient content. In addition, participants were asked

132 to remain inactive the day before each main trial and wore a uniaxial accelerometer 
133 (Lifecoder-EX; Suzuken Co Ltd, Nagoya, Japan) on the hip to monitor their daily

134 activity objectively during this period. The accelerometer defined 11 levels of activity

135 intensity $(0,0.5$, and $1-9)$, with 0 indicating the lowest intensity and 9 being the highest

136 intensity. A level of 4 corresponds to an intensity of $\sim 3$ metabolic equivalents [15]. In

137 addition, total step count (steps per day) was recorded and calculated from the

138 accelerometer using computerised software (Lifelyzer 05 Coach; Suzuken Co Ltd).

\section{Experimental design and protocol}

141 Participants underwent three, one-day laboratory-based trials in a random order: 1)

142 control, 2) accumulated walking and 3) accumulated walking with energy replacement.

143 The interval between trials was at least 7 days. A schematic illustration of the study

144 protocol is shown in Figure 1.

145

146

Insert Fig. 1 near here

147

148 Control trial. Participants reported to the laboratory at $0845 \mathrm{~h}$ after a 10 -h overnight fast

149 (no food or drink except water). Then, body mass was measured to the nearest $0.1 \mathrm{~kg}$

150 using a digital scale (Inner Scan 50; Tanita Corporation, Tokyo, Japan). After a 15-min 
151 rest, a fasting venous blood sample was collected in a seated position by venipuncture at

$1520 \mathrm{~h}(0900 \mathrm{~h})$. Participants then consumed a standardised meal for breakfast and rested

153 thereafter in a seated position (reading or writing) for $8 \mathrm{~h}(1700 \mathrm{~h})$. A second test meal

154 was consumed for lunch $3 \mathrm{~h}$ after the initiation of breakfast. Further venous blood

155 samples were collected by venipuncture at $2 \mathrm{~h}(1100 \mathrm{~h}), 4 \mathrm{~h}(1300 \mathrm{~h}), 6 \mathrm{~h}(1500 \mathrm{~h})$, and

$1568 \mathrm{~h}(1700 \mathrm{~h})$ after the initiation of breakfast for the measurement of circulating

157 concentrations of TG, non-esterified fatty acids (NEFA), 3-hydroxybutyrate (3-OHB),

158 insulin and glucose. NEFA was measured as a surrogate marker of substrate delivery to

159 the liver for TG synthesis and 3-OHB as a marker of hepatic fatty acid oxidation

160 affecting TG secretion. Furthermore, we used measures of insulin and glucose as

161 indicators of carbohydrate metabolism, with the former also implicated in modulating

162 lipoprotein lipase activity, the rate-limiting enzyme for the hydrolysis of TG.

163

164 Accumulated walking. The accumulated walking trial was identical to the control trial

165 except participants were asked to complete $20 \times 1.5 \mathrm{~min}$ bouts of treadmill walking

166 throughout the day. The speed of walking was at a self-selected pace determined during

167 the preliminary visit. Treadmill walks were completed at 1000, 1015, 1030, 1045, 1115,

$1681130,1145,1315,1330,1345,1400,1415,1430,1445,1515,1530,1545,1600,1615$, 
169 and $1630 \mathrm{~h}$. Most walking bouts were separated by $15 \mathrm{~min}$ of seated rest but three

170 longer breaks were incorporated to allow for blood sampling and consumption of lunch.

171 This timing was chosen because we have shown that a similar pattern of walking can

172 reduce postprandial TG in healthy, older women [16] and older women with

173 hypertriglyceridaemia [17]. Heart rate was monitored throughout the accumulated

174 walking bouts using short-range telemetry (Polar RCX3, Polar Electro, Kempele,

175 Finland), and ratings of perceived exertion (RPE) were assessed periodically [18].

176

177 Accumulated walking with energy replacement. The accumulated walking with energy

178 replacement trial was identical to the accumulated walking trial except that the gross

179 energy expended during accumulated walking (estimated during the preliminary visit)

180 was provided at breakfast. The exercise-induced gross energy expenditure was replaced

181 using white bread and was prescribed individually $(0.62 \pm 0.12 \mathrm{MJ}(149 \pm 28 \mathrm{kcal}), 2.4$

$182 \pm 0.4 \mathrm{~g}$ fat, $27.6 \pm 5.1 \mathrm{~g}$ carbohydrate, $4.5 \pm 0.8 \mathrm{~g}$ protein).

\section{Test meals}

185 Breakfast consisted of white bread with butter, a salad (made of lettuce, tomato, and

186 ham with an Italian dressing), scrambled egg (one egg with tomato ketchup and soybean 
187 oil), soup (made of whole milk with corn soup powder), apple, and yoghurt. In the 188 control and accumulated walking trials, breakfast provided $0.34 \mathrm{~g}$ fat, $1.15 \mathrm{~g}$ 189 carbohydrate, $0.35 \mathrm{~g}$ protein, and $38 \mathrm{~kJ}$ (9 kcal) energy per kilogram of body mass. In 190 the accumulated walking with energy replacement trial, breakfast provided $0.39 \pm 0.01$

$191 \mathrm{~g}$ fat, $1.68 \pm 0.14 \mathrm{~g}$ carbohydrate, $0.44 \pm 0.02 \mathrm{~g}$ protein, and $50 \pm 3 \mathrm{~kJ}(12 \pm 1 \mathrm{kcal})$ 192 energy per kilogram of body mass including replacement of the estimated gross energy 193 expenditure induced by exercise. For the control and accumulated walking trials, mean 194 macronutrient content of the breakfast was $18.2 \pm 2.4 \mathrm{~g}$ fat, $61.5 \pm 8.2 \mathrm{~g}$ carbohydrate, 195 and $18.7 \pm 2.5 \mathrm{~g}$ protein, which provided $2.0 \pm 0.3 \mathrm{MJ}$ (486 $\pm 65 \mathrm{kcal})$ energy (34\% 196 from fat, 51\% from carbohydrate, and 15\% from protein). For the accumulated walking 197 with energy replacement trial, mean macronutrient content of the breakfast was $20.6 \pm$ $1982.4 \mathrm{~g}$ fat, $89.1 \pm 9.0 \mathrm{~g}$ carbohydrate, and $23.2 \pm 2.5 \mathrm{~g}$ protein, which provided $2.7 \pm 0.3$

199 MJ $(635 \pm 67 \mathrm{kcal})$ energy $(29.1 \pm 0.9 \%$ from fat, $56.2 \pm 1.1 \%$ from carbohydrate, and

$200 \quad 14.6 \pm 0.2 \%$ from protein). Lunch consisted of a typical Japanese fish dish (made from

201 grilled salmon), a bowl of white rice, soup (made with soybean curd, seaweed, soybean

202 paste, and deep-fried soybean curd), and steamed vegetables (cabbage, carrot, cucumber,

203 potato, and broccoli with a mayonnaise dressing) with ham and a cream cracker. It 204 provided $0.34 \mathrm{~g}$ fat, $1.10 \mathrm{~g}$ carbohydrate, $0.35 \mathrm{~g}$ protein, and $38 \mathrm{~kJ}$ (9 kcal) energy per 
205 kilogram of body mass in all trials. Mean macronutrient content of the lunch was $18.7 \pm$

$2062.5 \mathrm{~g}$ fat, $59.8 \pm 7.8 \mathrm{~g}$ carbohydrate, and $18.2 \pm 2.4 \mathrm{~g}$ protein, which provided $2.0 \pm 0.3$

207 MJ (486 $\pm 65 \mathrm{kcal})$ energy (35\% from fat, 50\% from carbohydrate, and 15\% from

208 protein). Participants were asked to consume each test meal within $30 \mathrm{~min}$, and

209 consumption time was recorded and replicated in subsequent trials. Mean time to

210 consume breakfast and lunch was $20.1 \pm 3.7$ and $16.2 \pm 5.9 \mathrm{~min}$, respectively. None of

211 the participants reported nausea or any gastrointestinal discomfort during or after either

212 meal. Participants consumed water ad libitum during the first trial, and the pattern and

213 volume ingested was replicated in subsequent trials. Average water intake was $904 \pm$

$214332 \mathrm{~mL}$ over $8 \mathrm{~h}$.

215

216 Analytical methods

217 For serum TG, NEFA and 3-OHB measurements, venous blood samples were collected

218 into tubes containing clotting activators for isolation of serum (Venoject 2; Terumo

219 Corporation, Tokyo, Japan). Thereafter, samples were allowed to clot for $30 \mathrm{~min}$ at

220 room temperature and then centrifuged at $1861 \mathrm{~g}$ for $10 \mathrm{~min}$ at $4^{\circ} \mathrm{C}$. Serum was removed,

221 divided into aliquots, and stored at $-80^{\circ} \mathrm{C}$ for later analysis. For plasma insulin

222 measurements, venous blood samples were collected into tubes containing dipotassium 
223 salt-EDTA (Venoject 2; Terumo Corporation, Tokyo, Japan). For plasma glucose

224 measurements, venous blood samples were collected into tubes containing sodium

225 fluoride-EDTA (Venoject 2; Terumo Corporation, Tokyo, Japan). Thereafter, both tubes

226 were immediately centrifuged and the plasma supernatant was stored at $-80^{\circ} \mathrm{C}$ for later

227 analysis. Enzymatic, colorimetric assays were used to measure serum TG (Pure Auto S

228 TG-N; Sekisui Medical Co Ltd, Tokyo, Japan), serum NEFA (NEFA-HR; Wako Pure

229 Chemical Industries, Ltd, Osaka, Japan), serum 3-OHB (KAINOS 3-HB; Kainos

230 Laboratories, Inc, Tokyo, Japan), and plasma glucose (GLU-HK(M); Shino-Test

231 Corporation, Kanagawa, Japan). Enzyme-linked immunosorbent assays (ELISA) were

232 used to measure plasma insulin (Mercodia Insulin ELISA; Mercodia AB, Uppsala,

233 Sweden). Samples for each participant were analysed within the same run for each

234 measure. Intra-assay coefficients of variation were $0.7 \%$ for TG, $0.8 \%$ for NEFA, $0.7 \%$

235 for 3-OHB, $4.3 \%$ for insulin, and $0.7 \%$ for glucose.

236

237 Statistical analysis

238 Data were analysed using the Predictive Analytics Software (PASW) version 25.0 for

239 Windows (IBM SPSS Statistics 25.0, SPSS Japan Inc., Japan). The Shapiro-Wilk test

240 was used to check for normality of distribution - all parameters were found to be 
241 normally distributed. Time-averaged total area under the serum or plasma concentration

242 versus time curves (AUC) were calculated using the trapezium rule. Repeated-measures

243 one-factor analysis of variance (ANOVA) was used to assess differences among the

244 three trials for fasting serum or plasma concentrations, AUC values, and dietary and

245 physical activity data. Repeated measures, two-factor ANOVA was used to examine

246 differences over time among the three trials for serum TG. Repeated measures

247 generalised estimating equations were performed as a sensitivity analysisto examine

248 between-trial differences in the time-averaged AUC values with the respective fasting

249 serum or plasma concentration modeled as a covariate. Where significant trial-by-time

250 interactions and trial effects were found, the data were subsequently analysed using

251 post-hoc analysis and were adjusted for multiple comparisons using the Bonferroni

252 method. The $95 \%$ confidence interval $(95 \% \mathrm{CI})$ for the mean absolute pairwise

253 differences between the trials was calculated using the t-distribution and degrees of

254 freedom (n - 1). Absolute standardised effect sizes (ES) are provided to supplement the

255 findings. An ES of 0.2 was considered the minimum important difference in all outcome

256 measurements, 0.5 moderate and 0.8 large [19]. Data are expressed as means \pm SD.

257 Statistical significance was set at $p<0.05$. 
260 Dietary and body mass data

261 Mean self-reported energy intake for the day prior to each trial was $7.5 \pm 2.0 \mathrm{MJ}$ (1796

$262 \pm 457 \mathrm{kcal})$. Energy intake equated to $18 \pm 5 \%(67.4 \pm 29.7 \mathrm{~g} /$ day $)$ from fat, $61 \pm 5 \%$

$263(221.9 \pm 58.8 \mathrm{~g} /$ day $)$ from carbohydrate and $21 \pm 5 \%(74.9 \pm 16.8 \mathrm{~g} /$ day $)$ from protein.

264 Body mass did not differ on the morning of each main trial $(53.2 \pm 6.8$ vs $53.0 \pm 7.0$ vs

$26552.7 \pm 6.9 \mathrm{~kg}$ for the control, accumulated walking and accumulated walking with

266 energy replacement trials respectively; $\mathrm{ES}=0.081$, main effect of trial $p=0.256$ ).

267

268 Physical activity data

269 The step counts recorded the day before the trials did not differ across trials (8426 \pm

2703844 vs $8511 \pm 3644$ vs $7981 \pm 3197$ steps per day for the control, accumulated walking

271 and accumulated walking with energy replacement trials respectively; $\mathrm{ES}=0.017$, main

272 effect of trial $p=0.755)$. Accelerometer recorded frequencies for light (levels 1-3; $63 \pm$

27330 vs $62 \pm 22$ vs $54 \pm 26 \mathrm{~min} /$ day for the control, accumulated walking and accumulated

274 walking with energy replacement trials, respectively; $\mathrm{ES}=0.123$, main effect of trial $p$

$275=0.159$ ), moderate (levels $4-6 ; 31 \pm 22$ vs $28 \pm 19$ vs $22 \pm 18 \mathrm{~min} /$ day for the control,

276 accumulated walking and accumulated walking with energy replacement trials, 
277 respectively; $\mathrm{ES}=0.211$, main effect of trial $p=0.105$ ), and vigorous (levels 7-9; $2 \pm 2$

278 vs $2 \pm 1$ vs $2 \pm 3 \mathrm{~min} /$ day for the control, accumulated walking and accumulated

279 walking with energy replacement trials, respectively; $\mathrm{ES}=0.116$, main effect of trial $p$

$280=0.414)$ activity did not differ across trials.

281

282 Responses during accumulated walking

283 Self-selected brisk walking speed during the walking trials was $4.1 \pm 1.0 \mathrm{~km} / \mathrm{h}$. The

284 mean heart rate and RPE did not differ between the accumulated walking and

285 accumulated walking with energy replacement trials (heart rate, $91 \pm 12$ vs $90 \pm 12$

286 beats/min, respectively; $95 \% \mathrm{CI},-2$ to 4 beats $/ \mathrm{min}, \mathrm{ES}=0.243, p=0.546 ; \mathrm{RPE}, 10 \pm 1$

287 vs $10 \pm 1$, respectively; $95 \% \mathrm{CI},-3$ to $1, \mathrm{ES}=0.243, p=0.397$ ).

289 Fasting serum/plasma concentrations

290 Fasting plasma and serum concentrations for each trial are shown in Table 2. There

291 were no differences across trials in serum TG, NEFA, 3-OHB, and plasma insulin and

292 glucose concentrations (main effect of trial $p \geq 0.128$ ). 


\section{Postprandial serum/plasma concentrations}

297 Serum TG concentrations differed among trials (Fig. 2) $(\mathrm{ES}=0.226$, main effect of trial $298 p=0.017)$ and were lower in the accumulated walking than control trial $(95 \% \mathrm{CI}$ 2990.471 to $-0.320 \mathrm{mmol} / \mathrm{L}, p=0.022)$. However, the accumulated walking with energy 300 replacement trial was not different from the accumulated walking (95\% CI -0.043 to

$3010.325 \mathrm{mmol} / \mathrm{L}, p=0.172)$ or control $(95 \% \mathrm{CI}-0.364$ to $0.143 \mathrm{mmol} / \mathrm{L}, p=0.783)$ trials.

302 Post-hoc analysis of an interaction effect $(p=0.011)$ revealed that TG concentrations

303 were lower in the accumulated walking than control trial at $4 \mathrm{~h}(95 \% \mathrm{CI}-0.690$ to $304-0.075 \mathrm{mmol} / \mathrm{L}, p=0.013)$, and $8 \mathrm{~h}$ after breakfast $(95 \% \mathrm{CI}-0.591$ to $-0.056 \mathrm{mmol} / \mathrm{L}, p$ $305=0.016)$. Moreover, TG was lower with accumulated walking than accumulated 306 walking with energy replacement (95\% CI -0.442 to $-0.040 \mathrm{mmol} / \mathrm{L}, p=0.017)$ at $8 \mathrm{~h}$.

308 The time-averaged TG AUC (ES $=0.207$, main effect of trial $p=0.024)$ (Fig. 3) was

$30914 \%$ lower in the accumulated walking trial than the control trial $(95 \% \mathrm{CI}-0.491$ to

$310-0.022 \mathrm{mmol} / \mathrm{L} \mathrm{h}, p=0.030)$. Accumulated walking with energy replacement values for

311 time-averaged TG AUC did not differ from values in the accumulated walking (95\% CI

$312-0.057$ to $0.331 \mathrm{mmol} / \mathrm{L} \mathrm{h}, p=0.223)$ or control $(95 \% \mathrm{CI}-0.396$ to $0.157 \mathrm{mmol} / \mathrm{L} \mathrm{h}, p=$ 
313 0.795) trials. A sensitivity analysis using generalised estimating equations for the

314 time-averaged TG AUC to adjust for fasting TG concentrations also revealed a main

315 effect of trial $(p=0.004)$ with lower time-averaged TG AUC in the accumulated

316 walking than control trial $(95 \% \mathrm{CI}-0.423$ to $-0.009 \mathrm{mmol} / \mathrm{L}, p=0.003)$. However, the

317 accumulated walking with energy replacement trial was not different from the

318 accumulated walking $(95 \% \mathrm{CI}-0.275$ to $0.001 \mathrm{mmol} / \mathrm{L}, p=0.052)$ or control $(95 \% \mathrm{CI}$

$319-0.077$ to $0.316 \mathrm{mmol} / \mathrm{L}, p=0.234)$ trials. Please note that the results are similar to the

320 ANOVA and therefore do not alter the interpretation of the data.

321

Insert Fig. 2 near here

Insert Fig. 3 near here

325 The time-averaged AUC values for NEFA, 3-OHB, insulin, and glucose are shown in

326 Table 3. For NEFA (ES $=0.837$, main effect of trial $p<0.0005)$ the AUC was lower on

327 the accumulated walking with energy replacement trial than the control $(95 \% \mathrm{CI}-0.346$

328 to $-0.203 \mathrm{mmol} / \mathrm{L} \mathrm{h}, p<0.0005)$ and the accumulated walking $(95 \% \mathrm{CI}-0.381$ to -0.246

$329 \mathrm{mmol} / \mathrm{L} \mathrm{h}, p<0.0005)$ trials. The time-averaged 3-OHB and glucose AUC did not

330 differ among trials. The time-averaged insulin $(\mathrm{ES}=0.259$, main effect of trial $p=$ 
331 0.018) AUC was lower on the accumulated walking than control (95\% CI -26.47 to

$332-0.36 \mathrm{pmol} / \mathrm{L} \mathrm{h}, p=0.043)$ and accumulated walking with energy replacement $(95 \% \mathrm{CI}$

$333-51.67$ to $-2.31 \mathrm{pmol} / \mathrm{L} \mathrm{h}, p=0.030)$ trials. A sensitivity analysis using generalised

334 estimating equations for the time-averaged AUC values for NEFA, 3-OHB, insulin and

335 glucose to adjust for the respective fasting serum or plasma concentrations did not alter

336 the findings for any of the postprandial outcomes (data not shown). Mean values at each

337 time-point in the three trials for NEFA, 3-OHB, insulin and glucose concentrations are 338 provided in Supplementary Table 1. Insert Table 3 near here

\section{Discussion}

343 The present study demonstrated that accumulating short bouts of brisk walking (1.5 min

$344 \times 20)$ throughout the day reduced postprandial TG in older women but that dietary

345 replacement of the exercise-induced energy deficit diminished this effect. This

346 underscores the importance of maintaining an energy deficit in relation to exercise to

347 augment the reduction in postprandial TG even when exercise is accumulated in short

348 bouts during the day. 
350 The diminished postprandial TG-lowering effect of accumulated walking with

351 replacement of the exercise energy expenditure is consistent with previous studies in

352 adolescent boys [8], young men and women [6,7] and sedentary middle-age men [5]

353 when the postprandial measurements were conducted the day after exercise. The present

354 study extends these findings by demonstrating that the exercise-induced reduction in

355 postprandial TG is diminished on the same day exercise is performed, and this effect

356 persisted after adjusting for fasting concentrations. In contrast, other studies have

357 demonstrated that acute exercise reduced postprandial TG concentrations even when the

358 energy expended during exercise was replaced in young men and women [7, 9-11].

359 Differences in the intensity of exercise, and the composition and timing of the energy

360 replacement meals/drinks are possible reasons for the inconsistent findings among

361 studies. Since the intensity of exercise influences substrate partitioning during exercise

362 [20], the macronutrient composition of the energy replacement meal/drink may also

363 influence the subsequent postprandial TG response [9, 10]. Previous studies by Chiu

364 and colleagues [9] suggested that the exercise-induced fat deficit may be important,

365 whereas Trombold and colleagues [10] suggested the carbohydrate deficit may be

366 important in determining the reduction in postprandial TG after exercise. Alternatively, 
367 another study has suggested that the type of carbohydrate, low or high glycemic index,

368 is also an important factor [11]. It was not possible to isolate whether the

369 exercise-induced substrate deficit or the energy deficit per se were more important for

370 determining the reduction in postprandial TG in the present study and further work is

371 required to differentiate this [4].

372

373 The present findings suggest that the effect of exercise on postprandial TG

374 concentrations in these older women is dependent on an exercise-evoked energy deficit,

375 at least on the same day exercise is performed. Nevertheless, it is important to note that

376 an acute exercise-induced energy deficit appears more potent for provoking a reduction

377 in postprandial TG concentrations than an isoenergetic diet-induced energy deficit [21,

378 22], suggesting the effect of exercise is not solely attributable to the resulting energy

379 deficit.

380

381 One unique feature of the present investigation which extends previous studies is that

382 the extra energy provided preceded exercise - at breakfast -, rather than being given as a

383 post-exercise energy replacement. The walking accumulated throughout the day was

384 unable to attenuate TG elevations brought about by this extra pre-exercise energy. The 
385 ecological value of performing the experiment in this manner can be debated. Some

386 individuals may compensate for an exercise-induced energy expenditure immediately

387 afterwards. However, it also seems feasible that some individuals may perform

388 compensatory eating beforehand e.g. eat a big breakfast when they realise that they have

389 an active day ahead. Thus, the present study has novel features related to the order of

390 energy intake that have not previously been addressed. Despite these arguments, the

391 issue of compensatory eating in response to acute exercise has been challenged [23].

393 The reduction in postprandial TG concentrations observed in the accumulated walking

394 trial may have been mediated via several mechanisms although it is difficult to ascertain

395 which was acting or dominant. Reduced secretion of hepatic very-low density

396 lipoproteins seems unlikely as postprandial serum 3-OHB concentrations, an indicator

397 of hepatic fatty acid oxidation, were similar among trials, suggesting TG incorporation

398 into these lipoproteins did not differ. Similarly, chylomicron appearance was unlikely

399 affected with walking as blood flow to the splanchnic organs is not greatly

400 compromised with moderate exercise [24]. The lower postprandial plasma insulin

401 concentrations in the accumulated walking trial may have reduced insulin-mediated

402 inhibition of skeletal muscle lipoprotein lipase activity and therefore enhanced TG 
403 clearance at this site [25]. This effect is unlikely to have occurred in the accumulated

404 walking with energy replacement trial where the extra energy intake with white bread

405 increased insulin concentrations. Finally, increased skeletal muscle blood flow during

406 and immediately after each accumulated walk may have enhanced TG clearance by

407 increasing exposure of lipoprotein lipase to TG-rich lipoproteins. This effect may have

408 been offset with higher TG concentrations in the energy replacement condition.

409

410 The reduction in postprandial TG after accumulating short bouts of walking is in accord

411 with previous investigations in older women [16, 17]. However, the present study is the

412 first to compare accumulating short bouts of walking with and without dietary

413 replacement of the exercise-induced energy deficit on postprandial TG in older women.

414 It is not known whether this population would regularly engage in this intermittent brisk

415 walking pattern outside the laboratory setting. Nonetheless, we have previously found

416 that encouraging older postmenopausal women to increase their weekend engagement in

417 moderate-to-vigorous physical activity (average increase of $16 \mathrm{~min}$ in total) reduced

418 postprandial TG concentrations under free-living conditions [26]. Moreover, another

419 study has shown that middle-aged Japanese women typically engage in

420 moderate-to-vigorous physical activity bouts lasting $<3 \min$ [13]. Thus, the activity 
421 pattern adopted in the present study has ecological relevance and may be attractive to

422 many older adults as it can be easily incorporated into their day-to-day lives.

423 Furthermore, although the clinical relevance of the present study is not known, it has

424 been demonstrated recently that total moderate-to-vigorous physical activity of any bout

425 duration is associated with a lower risk of all-cause mortality [27]. Nonetheless, it

426 would be of future interest to examine the longer-term effects of accumulating short

427 bouts of daily physical activity on postprandial TG and other clinical outcomes in a

428 larger population.

429

430 The present study has several strengths. First, most studies examining the effects of

431 exercise on postprandial TG have employed young individuals with few studies in

432 individuals over 40 years (for a review of these, see [3]). The population recruited to the

433 present study is important since a substantial increase in the risk of cardiovascular

434 disease has been observed with higher concentrations of non-fasting TG and with

435 increasing age [2]. Furthermore, in many Asian countries, like Japan, recent temporal

436 shifts toward top-heavy elderly population structures [28] means that lifestyle

437 interventions shown to mitigate disease risk in older adults are becoming more critical.

438 Second, test meals of moderate-fat content (35\% of total energy) were used to assess 
postprandial metabolism in response to exercise with and without dietary replacement

440 of the exercise-induced energy deficit. Most studies in this field have employed high-fat

441 test meals (over $60 \%$ of total energy) (for a review of these, see [29, 30]). Thus, our test

442 meals mimic real life settings where the macronutrient composition of the test meals

443 reflects the background diet consumed in approximately one-third of Japanese adults

444 (34\% of Japanese women aged over 60 years consume daily meals containing over

$44530 \%$ of energy as fat) [31].

446

447 There are limitations to the experimental design employed. Expired air samples were

448 not collected to quantify directly the energy expenditure during the walking trials for

449 practical reasons given the short duration of the exercise bouts. This absence of energy

450 expenditure quantification may under- or over-estimate replacement of the energy

451 deficit in the accumulated walking with energy replacement trial. Furthermore, the

452 replacement of the exercise gross energy expenditure may have overestimated the

453 exercise-induced energy deficit. However, the difference between the net and gross

454 exercise energy expenditure is likely to be relatively small given the short duration and

455 low intensity of the exercise stimulus. 
457 In conclusion, accumulating $30 \mathrm{~min}$ of brisk walking in short (1.5 min) bouts reduced

458 postprandial TG but this effect was diminished when the exercise-induced energy

459 expenditure was replaced in older postmenopausal women. The findings of the present

460 study highlight the importance of maintaining an exercise-induced energy deficit on

461 exercise days to maximise the reduction in postprandial TG in older postmenopausal

462 women.

463

464 Acknowledgements

465 This study was supported by the Japan Society for the Promotion of Science

466 (No.17K01874, 2017-2019), the Japan Society for the Promotion of Science

467 Core-to-Core Program, A. Advanced Research Networks and Waseda University Grant

468 for Special Research Projects (2018K-370, 2018-2019). Alice Thackray and David

469 Stensel acknowledge support from the National Institute for Health Research (NIHR)

470 Leicester Biomedical Research Centre. The views expressed are those of the authors

471 and not necessarily those of the NHS, the NIHR, or the Department of Health.

472

\section{Author contributions}

474 M. M. conceived the study, obtained the funding and took the lead in writing the 


\section{Compliance with ethical standards}

\section{$481 \quad$ Ethical standard}

482 This study was approved by the Ethics Committee on Human Research of Waseda 483 University (Approval number: 2017-103). Written informed consent was obtained from 484 all participants prior to the study.

\section{Conflict of interest}

487 All authors declare that there is no conflict of interest.

488

\section{References}

490 1. Iso H, Imano H, Yamagishi K, Ohira T, Cui R, Noda H, Sato S, Kiyama M, Okada

491 T, Hitsumoto S, Tanigawa T, Kitamura A, CIRCS Investigators (2014) Fasting and non-fasting triglycerides and risk of ischemic cardiovascular disease in Japanese 
men and women: the Circulatory Risk in Communities Study (CIRCS).

494 Atherosclerosis 237(1):361-368. https://doi: 10.1016/j.atherosclerosis.2014.08.028.

495 2. Nordestgaard BG, Varbo A (2014) Triglycerides and cardiovascular disease. Lancet 384(9943):626-635. https://doi: 10.1016/S0140-6736(14)61177-6.

497 3. Freese EC, Gist NH, Cureton KJ (2014) Effect of prior exercise on postprandial 498 lipemia: an updated quantitative review. J Appl Physiol (1985) 116(1):67-75. 499 https://doi: 10.1152/japplphysiol.00623.2013.

500 4. Braun B, Brooks GA (2008) Critical importance of controlling energy status to 501 understand the effects of "exercise" on metabolism. Exerc Sports Sci Rev 36(1):2-4. https://doi: 10.1097/jes.0b013e31815e42c2.

5. Burton FL, Malkova D, Caslake MJ, Gill JMR (2008) Energy replacement attenuates the effects of prior moderate exercise on postprandial metabolism in overweight/obese men. Int J Obes 32(3):481-489. https://doi: 10.1038/sj.ijo.0803754.

6. Harrison M, O'Gorman DJ, McCaffrey N, Hamilton MT, Zderic TW, Carson BP, Moyna NM (2009) Influence of acute exercise with and without carbohydrate replacement on postprandial lipid metabolism. J Appl Physiol (1985) 106(3):943-949. https://doi: 10.1152/japplphysiol.91367.2008. 
511 7. Freese EC, Levine AS, Chapman DP, Hausman DB, Cureton KJ (2011) Effects of

512 acute sprint interval cycling and energy replacement on postprandial lipemia. J

$513 \quad$ Appl Physiol (1985) 111(6):1584-9. https://doi: 10.1152/japplphysiol.00416.2011.

514 8. Thackray AE, Barrettt LA, Tolfrey K. (2016) Energy replacement diminishes the

515 effect of exercise on postprandial lipemia in boys. Metabolism 65(4):496-506.

516 https://doi: 10.1016/j.metabol.2015.12.001.

517 9. Chiu CH, Burns SF, Yang TJ, Chang YH, Chen YL, Chang CK, Wu CL (2014)

518 Energy replacement using glucose does not increase postprandial lipemia after

519 moderate intensity exercise. Lipids Health Dis 13:177. https://doi:

$520 \quad 10.1186 / 1476-511 X-13-177$.

521 10. Trombold JR, Christmas KM, Machin DR, Van Pelt DW, Chou TH, Kim IY, Coyle

522 EF (2014) Postexercise macronutrient intake and subsequent postprandial

523 triglyceride metabolism. Med Sci Sports Exerc 46(11):2099-2106. https://doi:

$524 \quad$ 10.1249/MSS.0000000000000333.

525 11. Kaviani M, Chilibeck PD, Yee P, Zello GA (2016) The effect of consuming low-

526 versus high-glycemic index meals after exercise on postprandial blood lipid

527 response following a next-day high-fat meal. Nutr Diabetes 6(7):e216. https://doi:

$528 \quad 10.1038 /$ nutd.2016.26. 

Diurnal triglyceride profiles: a novel approach to study triglyceride changes. Atherosclerosis 155(1):219-228. short bouts of non-exercise physical activity associated with metabolic syndrome under free-living conditions in Japanese female adults. Eur J Appl Physiol 112(10): 3525-3532. https://doi: 10.1007/s00421-012-2342-8.

14. Weir JB (1949) New methods for calculating metabolic rate with special reference to protein metabolism. J Physiol 109(1-2):1-9. https://doi. 10.1113/jphysiol.1949.sp004363.

15. Kumahara H, Schutz Y, Ayabe M, Yoshioka M, Yoshitake Y, Shindo M, Ishii K, Tanaka H (2004) The use of uniaxial accelerometry for the assessment of physical-activity-related energy expenditure: a validation study against whole-body indirect calorimetry. Br J Nutr 91(2):235-243. https://doi: 10.1079/BJN20031033. Burns S (2016) Interrupting sitting time with regular walks attenuates postprandial triglycerides. Int J Sports Med 37(2):97-103. https://doi: 10.1055/s-0035-1559791. 
18. Borg GA (1973) Perceived exertion: a note on "history" and methods. Med Sci Sports 5(2):90-93.

19. Cohen J (1988) Statistical Power Analysis for the Behavioural Sciences, 2nd edn. Lawrence Erlbaum Associates: Hillsdale, NJ, USA.

20. Coyle EF (1995) Substrate utilization during exercise in active people. Am J Clin Nutr 61(4 Supplement):968S-979S. https://doi. 10.1093/ajcn/61.4.968S.

21. Gill JM, Hardman AR (2000) Postprandial lipemia: effects of exercise and restriction of energy intake compared. Am J Clin Nutr 71(2):465-471. https://doi.10.1093/ajen/71.2.465.

22. Thackray AE, Barrett LA, Tolfrey K (2015) Acute effects of energy deficit induced in healthy girls. Pediatr Exerc Sci 27(2):192-202. https://doi.10.1123/pes.2014-0096. 
modulating effect of adiposity, sex, and habitual physical activity. Nutrients 10(9):E1140. https//doi: 10.3390/nu10091140.

567 24. Eriksen M, Waaler BA (1994) Priority of blood flow to splanchnic organs in 568 humans during pre- and post-meal exercise. Acta Physiol Scand 150(4):363-372. https://doi. 10.1111/j.1748-1716.1994.tb09700.x.

25. Seip RL, Semenkovich CF (1998) Skeletal muscle lipoprotein lipase: Molecular regulation and physiological effects in relation to exercise. Exerc Sport Sci Rev 26(1):191-218.

26. Miyashita M, Takahashi M, Burns SF (2014) Increased participation in weekend physical activity reduces postprandial lipemia in postmenopausal women. Int J Sports Med 35(13):1059-1064. https://doi: 10.1055/s-0034-1372641.

27. Saint-Maurice PF, Troiano RP, Matthews CE, Kraus WE (2018) Moderate-to-vigorous physical activity and all-cause mortality: Do bouts matter? J Am Heart Assoc 7(6): e007678. https://doi.org/10.1161/JAHA.117.007678. Prospects 2019: Highlights. United Nations: New York, 2019. https://population.un.org/wpp/Publications/Files/WPP2019_Highlights.pdf 
583 29. Gill JMR, Hardman AE (2003) Exercise and postprandial lipid metabolism: an

584 update on potential mechanisms and interactions with high-carbohydrate diets

585 (review).

Nutr

Biochem

$14(3): 122-132$.

586

https://doi.org/10.1016/S0955-2863(02)00275-9.

587 30. Petitt DS, Cureton KJ. Effects of prior exercise on postprandial lipemia: a

588

quantitative

review.

Metabolism

52(4):418-424.

589 https://doi.org/10.1053/meta.2003.50071.

590

31. Ministry of Health, Labour and Welfare, Japan. The National Health and Nutrition Survey in Japan, 2017. Ministry of Health, Labour and Welfare: Tokyo, 2018. https://www.mhlw.go.jp/content/000451755.pdf (Accessed: 1 July 2019) 
594 Fig. 1 A schematic representation of the study protocol.

595 For the control trial, participants sat in a chair (reading and writing) in the laboratory

596 between $0 \mathrm{~h}(0900 \mathrm{~h})$ and $8 \mathrm{~h}(1700 \mathrm{~h})$. For the accumulated walking and accumulated

597 walking with energy replacement trials, participants rested for 40 minutes after 598 consuming breakfast before performing twenty, 1.5-min bouts of brisk walking on a 599 treadmill throughout the day. Rep.; replacement. $0.62 \pm 0.12 \mathrm{MJ}(149 \pm 28 \mathrm{kcal})$.

600

601 Fig. 2 Fasting and postprandial serum triglyceride (TG) concentrations during the 602 control, accumulated walking and accumulated walking with energy replacement 603 trials.

604 Data are means \pm SD for $n=17$. The black rectangles indicate the times that the test 605 meals were consumed. Data were analysed using 2-factor ANOVA. Post-hoc analysis 606 was adjusted for multiple comparisons using the Bonferroni method. There was a 607 significant main effect of trial $(p=0.017)$, main effect of time $(p<0.0005)$ and trial $\times$ 608 time interaction $(p=0.011) .{ }^{*}$ Significantly different between accumulated walking and 609 control trials. ${ }^{\dagger}$ Significantly different between accumulated walking and accumulated 610 walking with energy replacement trials. 
612 Fig. 3 The time-averaged serum triglyceride (TG) area under the curve (AUC)

613 values over $8 \mathbf{h}$ after the consumption of the test meals in the control, accumulated

614 walking and accumulated walking with energy replacement trials.

615 Data are means \pm SD for $\mathrm{n}=17$. Means were compared using one-factor ANOVA.

616 Post-hoc analysis was adjusted for multiple comparisons using the Bonferroni method.

617 Significantly different from the control trial, $p<0.05$. 
618 Table 1. Physical characteristics of the 17 participants.

\begin{tabular}{lc}
\hline Characteristic & Mean (SD) \\
\hline Age (years) & $70.2(2.9)$ \\
Height (m) & $1.55(0.05)$ \\
Body mass (kg) & $53.5(7.1)$ \\
Body mass index $\left(\mathrm{kg} / \mathrm{m}^{2}\right)$ & $22.3(3.0)$ \\
Waist circumference $(\mathrm{cm})$ & $79.2(9.2)$ \\
Systolic blood pressure $(\mathrm{mmHg})$ & $133(18)$ \\
Diastolic blood pressure $(\mathrm{mmHg})$ & $81(11)$ \\
\hline
\end{tabular}


619 Table 2. Fasting concentrations of triglyceride (TG), non-esterified fatty acids (NEFA), 3-hydroxybutyrate (3-OHB), insulin and

620 glucose in the control, accumulated walking and accumulated walking with energy replacement trials.

\begin{tabular}{|c|c|c|c|c|c|c|}
\hline Trial & Control & $\begin{array}{l}\text { Accumulated } \\
\text { walking }\end{array}$ & $\begin{array}{l}\text { Accumulated } \\
\text { walking with } \\
\text { energy } \\
\text { replacement }\end{array}$ & $\begin{array}{l}\text { Control vs accumulated } \\
\text { walking } \\
95 \% \mathrm{CI}^{\mathrm{a}}\end{array}$ & $\begin{array}{l}\text { Control vs accumulated } \\
\text { walking with energy } \\
\text { replacement } \\
95 \% \mathrm{CI}^{\mathrm{a}}\end{array}$ & $\begin{array}{c}\text { Accumulated walking with } \\
\text { energy replacement vs } \\
\text { accumulated walking } \\
95 \% \mathrm{CI}^{\mathrm{a}}\end{array}$ \\
\hline $\begin{array}{c}\mathrm{TG} \\
(\mathrm{mmol} / \mathrm{L})\end{array}$ & $1.06(0.60)$ & $0.95(0.59)$ & $1.02(0.56)$ & -0.26 to 0.03 & -0.19 to 0.11 & -0.21 to 0.07 \\
\hline $\begin{array}{c}\text { NEFA } \\
(\mathrm{mmol} / \mathrm{L})\end{array}$ & $0.62(0.22)$ & $0.65(0.27)$ & $0.64(0.25)$ & -0.11 to 0.18 & -0.11 to 0.15 & -0.10 to 0.13 \\
\hline $\begin{array}{c}3-\mathrm{OHB} \\
(\mathrm{mmol} / \mathrm{L})\end{array}$ & $0.10(0.08)$ & $0.11(0.12)$ & $0.11(0.10)$ & -0.05 to 0.07 & -0.06 to 0.07 & -0.05 to 0.06 \\
\hline $\begin{array}{c}\text { Insulin } \\
(\mathrm{pmol} / \mathrm{L})\end{array}$ & $21.6(11.5)$ & $22.8(15.1)$ & $25.4(20.0)$ & -6.44 to 8.77 & -4.60 to 12.06 & -10.60 to 5.44 \\
\hline $\begin{array}{l}\text { Glucose } \\
(\mathrm{mmol} / \mathrm{L})\end{array}$ & $5.28(0.67)$ & $5.27(1.04)$ & $5.29(0.96)$ & -0.37 to 0.36 & -0.22 to 0.25 & -0.28 to 0.24 \\
\hline
\end{tabular}

622 Values are mean (SD) for $\mathrm{n}=17$. Means were compared using one-factor ANOVA. Analysis revealed no main effect of trial for any 
624 Table 3. The time-averaged serum non-esterified fatty acids (NEFA), 3-hydroxybutyrate (3-OHB), and plasma insulin and glucose area

625 under the curve (AUC) values over $8 \mathrm{~h}$ after the consumption of the test meals in the control, accumulated walking and accumulated

626 walking with energy replacement trials.

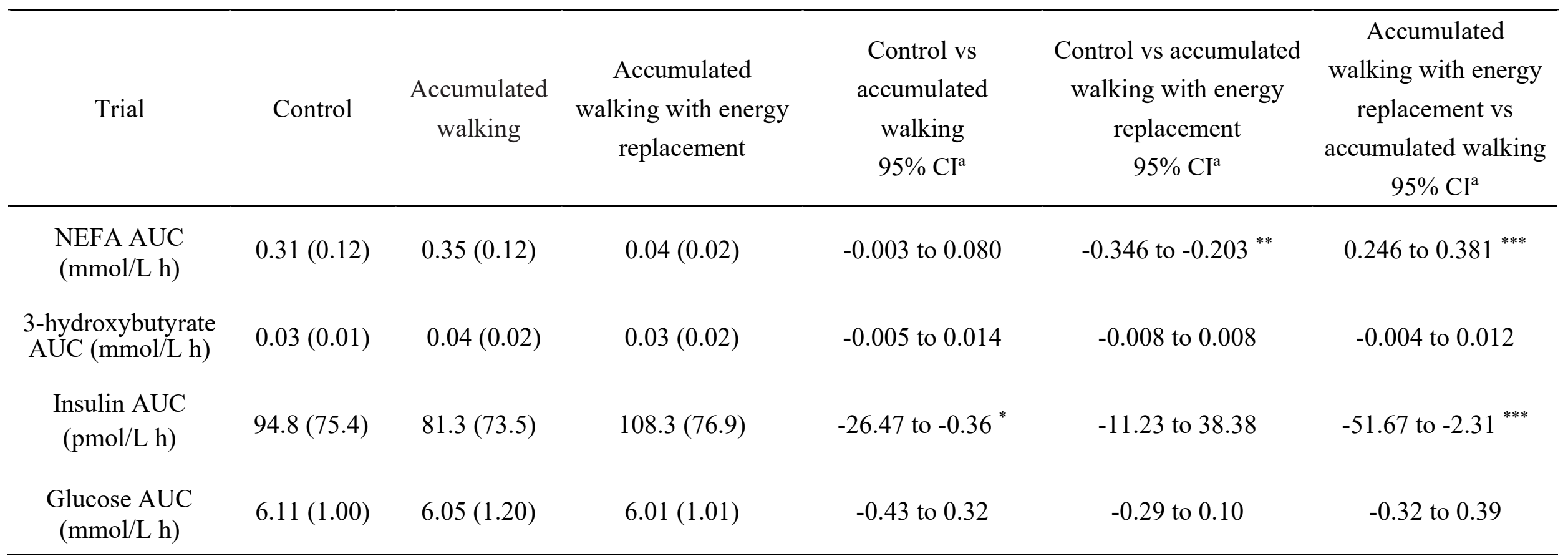

627 Values are mean (SD) for $\mathrm{n}=17$. Means were compared using one-factor ANOVA and post-hoc analysis was adjusted for multiple

628 comparisons using the Bonferroni method. ${ }^{a}$ 95\% confidence interval (CI) of the mean absolute difference between the experimental 
630 of trial: ${ }^{*} p<0.05$ between accumulated walking and control; ${ }^{* *} p<0.05$ between accumulated walking with energy replacement and

631 control; ${ }^{* * *} p<0.05$ between accumulated walking and accumulated walking with energy replacement. 